\title{
BLAISE CENDRARS, JACQUES-HENRY LÉVESQUE, Correspondance 1922-1959. «Et maintenant veillez au grain!»
}

\section{Riccardo Benedettini}

\section{(2) OpenEdition}

\section{Edizione digitale}

URL: http://journals.openedition.org/studifrancesi/15787

DOI: $10.4000 /$ studifrancesi. 15787

ISSN: 2421-5856

\section{Editore}

Rosenberg \& Sellier

\section{Edizione cartacea}

Data di pubblicazione: 1 décembre 2018

Paginazione: 527

ISSN: 0039-2944

\section{Notizia bibliografica digitale}

Riccardo Benedettini, «BLAISE CendRARS, JACQues-HenRy LÉvesque, Correspondance 1922-1959. «Et maintenant veillez au grain!»», Studi Francesi [Online], 186 (LXII | III) | 2018, online dal 01 janvier 2019, consultato il 06 janvier 2021. URL: http://journals.openedition.org/studifrancesi/15787 ; DOI: https://doi.org/ 10.4000/studifrancesi. 15787

Questo documento è stato generato automaticamente il 6 janvier 2021.

\section{(c) 98}

Studi Francesi è distribuita con Licenza Creative Commons Attribuzione - Non commerciale - Non opere derivate 4.0 Internazionale. 


\title{
BLAISE CENDRARS, JACQUES-HENRY LÉVESQUE, Correspondance 1922-1959. «Et maintenant veillez au grain!»
}

\author{
Riccardo Benedettini
}

\section{NOTIZIA}

BLAISE CENDRARS, JACQUES-HENRY LÉVESQUE, Correspondance 1922-1959. «Et maintenant veillez au grain!», texte établi, annoté et présenté par Marie-Paule Berranger, Carouge-Genève, Zoé, 2017, «Cendrars en toutes lettres», 752 pp.

Continuando l'eccellente pubblicazione della corrispondenza di Blaise Cendrars nella collana diretta da Christine Le Quellec Cottier, Marie-Paule Berranger, collaboratrice della recente edizione «Pléiade» delle Cuvres romanesques précédé de Poésies complètes dello scrittore svizzero naturalizzato francese, raccoglie in questo volume 37 anni di scambi epistolari: 666 lettere che Cendrars invia al discepolo Lévesque tra il 1922 e il 1958, e 74 lettere scritte dal giovane ammiratore al poeta. Come segnala Miriam Cendrars nell'«Avant-propos» (pp.5-6), le lettere del padre sono oggi in gran parte conservate presso il «Fonds Blaise Cendrars des Archives littéraires suisses»; le altre provengono invece dall'archivio privato della moglie di Lévesque, Angèle, tenuto conto che Cendrars non era solito conservare la posta ricevuta. Indubbiamente sul piano filologico la conoscenza del testo cendrarsiano ha fatto in questi ultimi anni dei passi notevoli e fruttuose sono state le ricerche intorno al problema dell'ordine secondo il quale devono essere classificate le sue memorie. La corrispondenza ora edita (e ciò vale anche per quella degli anni 1937-1954 con Raymone Duchâteau, a cura di M. Boucharenc, Zoé, 2015: cfr. questa rassegna n. 179, 2016, p. 361) è «presque complète» (p.12) e qui disposta secondo sette archi temporali: come leggiamo nella «Préface. J'écris comme un possédé...» (pp. 7-25), essa è nata «sous le signe de la spontanéité», senza «le souci de construire une image publique» (p.15). 
2 Nelle lettere ritroviamo il carattere privato, sempre cortese, che dovevano avere le conversazioni tra i due amici e collaboratori. Gli argomenti sono tra i più vari, tutti di estremo interesse per gli studiosi: dalle occasioni conviviali (come avvalorato dalle immagini presentate) alle richieste, ripetute negli anni, di libri che il "giramondo" non ha con sé (la sua Anthologie nègre, di cui Cendrars sembra aver sempre assoluto bisogno) o di ricerche da condurre nelle biblioteche; dalle riflessioni sulla scrittura alle difficoltà del vivere quotidiano («Et maintenant veillez au grain!», p. 414, suggerisce Lévesque allo scrittore che, proprio in queste lettere, si definisce per la prima volta «foudroyé», p. 120 e p.127); dalle formule, talvolta torbide, riservate ad amici, o forse nemici (Cocteau, Gide, Cingria...), a quelle su uomini quali Hitler e Mussolini; dai consigli su come dirigere una rivista ai pensieri sull'umanità (il suicidio, l'aviazione, la guerra, il sistema coloniale, le avanguardie); dai ricordi di viaggio alla simbologia cristica, fino alla «dislocation du temps» (p.359), di cui Cendrars è maître. Questo bel volume si chiude con due appendici- «Échos» (pp.693-703), con brouillons di lettere, e «Résonances» (pp. 705-741), con scritti di Lévesque-e un «Index» dei nomi (pp. 742-750). 